\title{
Determining Number of Independent Sources in Undercomplete Mixture
}

\author{
Ganesh R. Naik and Dinesh K. Kumar \\ School of Electrical and Computer Engineering, RMIT University, GPO Box 2476V, Melbourne, VIC 3001, Australia \\ Correspondence should be addressed to Ganesh R. Naik, ganesh.naik@rmit.edu.au
}

Received 14 March 2009; Revised 28 July 2009; Accepted 2 September 2009

Recommended by Shoji Makino

\begin{abstract}
Separation of independent sources using independent component analysis (ICA) requires prior knowledge of the number of independent sources. Performing ICA when the number of recordings is greater than the number of sources can give erroneous results. To improve the quality of separation, the most suitable recordings have to be identified before performing ICA. Techniques employed to estimate suitable recordings require estimation of number of independent sources or require repeated iterations. However there is no objective measure of the number of independent sources in a given mixture. Here, a technique has been developed to determine the number of independent sources in a given mixture. This paper demonstrates that normalised determinant of the global matrix is a measure of the number of independent sources, $N$, in a mixture of $M$ recordings. It has also been shown that performing ICA on $N$ randomly selected recordings out of $M$ recordings gives good quality of separation.
\end{abstract}

Copyright (c) 2009 G. R. Naik and D. K. Kumar. This is an open access article distributed under the Creative Commons Attribution License, which permits unrestricted use, distribution, and reproduction in any medium, provided the original work is properly cited.

\section{Introduction}

Blind Source Separation (BSS) consists of estimating the original signals $\mathbf{s}$, from a finite set of observations $\mathbf{x}$, when $\mathbf{x}$ is a result of mixing the original signals $\mathbf{s}$. The estimation is done without any prior knowledge of the sources (or components) or the transmitting media. Independent Component Analysis (ICA) [1-4] has become a widely accepted technique to solve the BSS problem with applications in medicine, communications, and image processing [5-7]. It is based on the assumption that the components are independent and immobile.

There are two major issues associated with BSS; (i) estimation of number of independent components (ICs) and (ii) separation of the ICs. For conceptual and computational simplicity, most ICA algorithms assume that the number of components, $N$, is equal to the number of recordings, $M$, and all of these components are independent. This results in a square mixing matrix. This simplifies the estimation of the mixing matrix $\mathbf{A}$ and the unmixing matrix $W$ because square matrices are invertible. However, for number of applications this assumption is not accurate. If $N$ is greater than $M$, then the situation is referred to as "over-complete" while when $N$ is less than $M$, it is referred to as "undercomplete." In the overcomplete situation, performing ICA can result in incorrect separation and poor quality [8], [9]. Bofill and Zibulevsky [10] have developed techniques to identify the number of ICs in a mixture for the over-complete situation. While undercomplete situation has not been studied extensively, it is often encountered for applications such as sensor networks where the numbers of sensors may often exceed the number of components such as in sensor networks for environmental or defense monitoring, or when the components are not independent $[9,11,12]$.

Standard ICA techniques assume $N=M$. In an undercomplete situation, $N<M$, but ICA algorithms are inherently based on the assumption that $N=M$ and the algorithm attempts to estimate more number of ICs than actually exist. This can result in poor quality of separation. To overcome this shortcoming, the number of recordings, $M$, has to be reduced prior to the use of ICA. One method for removing the redundant and dependent recordings is to perform principal component analysis (PCA) on the data matrix $X$ and retain the $N$ principal components (PCs) from $M$ recordings. This approach is based on prior assumption of the number of ICs to be $N$ which may be 
only a guess in the true BSS situation. An improvement to what previously mentioned has been proposed by Vrins et al. [13] and is called selection PCA (sPCA). In sPCA, PCA is preceded by selecting a subset of the mixtures from the available ones using mutual information criterion. Although the results using SPCA are better than using PCA, this does not overcome the fundamental issue which is the need for guessing the value of $N$. It requires the prior assumption of the number of ICs and incorrect assumption can lead to erroneous results.

Stone and Porrill [14] proposed an alternative approach based on the maximisation of joint entropy of the output and using correlation between output and input to recover the ICs for undercomplete situation. This technique is an iterative approach that minimises the correlation between the inputs and outputs. However it requires the user to identify a threshold of this value or assume the value of $N$. Using the iterative approach, one IC is extracted at a time until the correlation is below a prespecified threshold. This is obviously a slow process and requires manual supervision and not suitable for automation.

From the above, it is evident that for successful implementation of ICA when $M>N$ (undercomplete), the number of ICs has to be determined before attempting IC separation. This paper proposes a new measure to identify the number of ICs. Once the number of ICs, $N$, is known, and based on the assumption of stationary sources and linear mixing, randomly selecting $N$ recordings as an input to ICA is proposed to separate the ICs in an undercomplete situation. The technique is a two-step approach. In the first step ICA is performed on all the $M$ recordings to generate the global matrix, $G$. The determinant of this matrix is used for obtaining $K$, the estimate of the number of ICs, $N$, in the given $M$ recordings. The next step requires randomly selecting $K$ out of $M$ recordings and performing ICA on these $K$ recordings. A comparison of the experimentally determined $K$ with prior known $N$ indicates that the technique is a very good measure of the number of independent sources in the recordings. A comparison of this approach with the rank, $R$, of $G$ indicates that $R$ is not suitable for estimating $N$. The results also indicate that there is a significant improvement of quality of separation of the ICs when this approach is used.

\section{Global Matrix Parameters of ICA}

The hypothesis of this research is that the determinant of the Global matrix, $G$, based on ICA performed on the $M$ recordings can be used to identify dependence between the components. When using synthetic data, the mixing matrix, $A$, is known, and $G$ is the product of the mixing matrix $A$ and estimated unmixing matrix $W$, or $G=W * A$. In real situations, when $A$ is not known, $G$ can be estimated from the product of the unmixing matrix of subband, $p$ and the inverse of the unmixing matrix of subband, $q[6]$ :

$$
G_{p q}=W_{p} \times W_{\mathrm{q}}^{-1} .
$$

If the number of recordings, $M=N$ (number of ICs), and the separation is accurate, then $G$ will be sparse and in ideal case, $G=I$, unity matrix (but for ICA order ambiguity). The mixing matrix and the unmixing matrix would be square and of size $M \times M$. Based on the independence criterion, the $|G|$ (determinant of $G$ ) would be unity after normalization, $\|G\|$ $=1($ norm of $G)[15]$.

When $M>N$, then the mixing matrix is a rectangular matrix of size $M \times N$, denoted by $A_{1}$. However, when ICA estimates the unmixing matrix, it estimates a square matrix of size $M \times M$. This unmixing matrix, denoted by $W$, corresponds to the mixing matrix, $A$ of size $M \times M$. There are $(M-N)$ extra columns in the matrix, $A$ compared with $A_{1}$. The extra columns correspond to the nonexistent sources, $s_{i}$ and the elements of these columns will be zero. Because of the iterative nature of ICA, the elements of these columns will be approximately zero and not exactly zero. When a matrix has one or more zero columns, its determinant is zero, and hence the determinant of the mixing matrix $|A| \approx 0$. Since $G$ is a product of $W$ and $A$, and when

$$
|A| \approx 0 \Longrightarrow|G| \approx 0 .
$$

In real situations, the number of sources is not known, and only the number of recordings, $M$, is known. If $|G| \approx$ 0 indicates that either $|A| \approx 0$ or $|W| \approx 0 .|W| \approx 0$ is a trivial outcome and thus can be ignored, and hence $|G| \approx 0$ indicates $|A| \approx 0$. When $|A| \approx$, there is no true inverse of $A$ which means that there is no good estimate of $W$ and the estimation of $\mathbf{s}=\mathbf{A}^{-1} \mathbf{x}$ would be erroneous. The obvious outcome when ICA is used in such a situation would be that the quality of separation would be poor.

From the above, it can be stated that if $|G| \approx 0$, then $|A| \approx 0$. This indicates that one or more column of $|A|$ are zero columns, and the mixing matrix is rectangular matrix, $A_{1}$ of size $M \times N$, with $M \neq N$. Based on the earlier assumption that this is not an overcomplete issue, this indicates that $M>N$. Thus $|G| \approx 0$ indicates that $M>N$ while $|G| \approx 1$ would indicate that $M=N$. However, ICA is associated with scaling ambiguity. To overcome this, the normalized values of $|G|,\|G\|$ have been considered in this research.

Experimental results indicate that when $M=N$ (the number of sources and the number of recordings are same) $\|G\| \approx 1$ while when $M>N\|G\| \approx 0$. The results also indicate that $\|G\|$ becomes closer to zero with an increase in the difference, $M-N$. $\|G\|$ is an indicator of the number of ICs, with the smaller value of $\|G\|$ indicating less independence between the estimated sources, and if there is only one IC, $\|G\|$ is nearly zero. The experimental results indicate a close relationship of $\|G\|$ with the number of dependent components in a mixture and can be used to obtain $K$, the estimate of $N$ in the given $M$ number of recordings. $\|G\|$ falls is a narrow range for a given number of dependent sources in a mixture, and this range is not sensitive to the number of recordings $M$. A comparison of the experimentally obtained $K$ with $N$ indicates that $K$ is indeed a very good estimate of $N$.

The step mentioned above uses ICA to estimate the number of ICs, and another step is required to separate the ICs from the mixture. Based on the assumption of 
TABLE 1: Criterion of mixing sources to generate the four recordings.

\begin{tabular}{lll}
\hline Criterion & Sources & $\begin{array}{l}\text { Recordings for } \\
i=1 \text { to } M\end{array}$ \\
\hline $\begin{array}{l}M \text { independent } \\
\text { components (all } \\
\text { independent) }\end{array}$ & $\begin{array}{l}\text { Four } \\
\text { independent } \\
\text { audiofiles } \\
s_{1}, s_{2}, s_{3} \text {, and } s_{4} \\
(N=M)\end{array}$ & $\begin{array}{l}x_{i}= \\
a_{i, 1} s_{1}+a_{i, 2} s_{2}+ \\
a_{i, 3} s_{3}+a_{i, 4} s_{4}, \\
\text { for } i=1 \text { to } 4\end{array}$ \\
\hline $\begin{array}{l}M-1 \\
\text { independent }\end{array}$ & Three & $x_{i}=$ \\
$\begin{array}{l}\text { and one } \\
\text { dependent }\end{array}$ & audiofiles $s_{1}, s_{2}$, & $a_{i, 1} s_{1}+a_{i, 2} s_{2}+$ \\
components & and $s_{3}$ & $a_{i, 3} s_{3}+a_{i, 4} s_{1}$, for \\
(N) & $i=1$ to 4
\end{tabular}

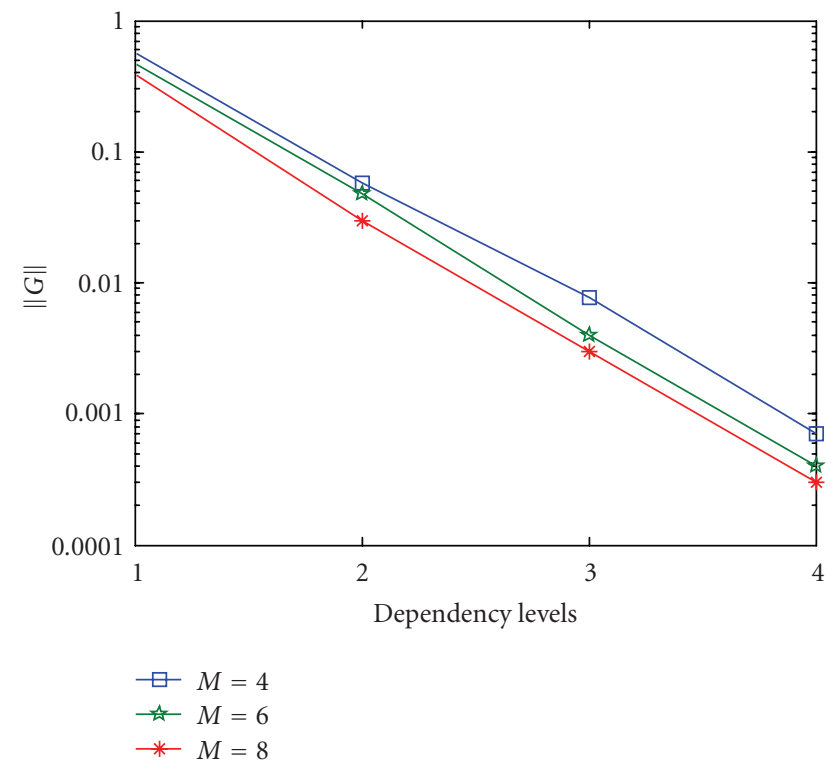

FIGURE 1: Logarithmic plot $\|G\|$ against dependency levels. Dependency level 1 corresponds with $N=M, 2$ corresponds with $N=$ $M-1,3$ corresponds with $N=M-2$, and 4 corresponds with $N=1$.

linearity of the mixing matrix, any (randomly selected) $K$ out of $M$ recordings should be suitable for estimating the original signals $\mathbf{s}$ without any need for assuming that all the components are independent. This eliminates the need to use PCA, correlation, or other methods to remove the redundant recordings. While this methodology is suitable for being used with all ICA techniques, this paper reports the use of FastICA $[3,16]$ to estimate the ICs from the mixture.

\section{Methodology}

Two sets of experiments were conducted. The first experiment was for determining the relationship between the $\|G\|$ and the number of ICs, $N$, given $M$ recordings, and $M>N$. The second experiment was conducted to test the hypothesis that once the number of ICs, $N$, is known, ICA can be used to separate any $N$ recordings to get good estimate of the original signals. Each experiment was repeated 10 times. The details of the experiments are given below.

Experiment 1. Experiments were conducted using $M$ synthetically mixed recordings according to Table 1 , with $M=$ four, six, and eight and with $N$ number of independent audiofiles, $N=$ two, four, six, and eight. Using FastICA, $M \times M$ global matrix, $G$, was generated and the $|G|$ was computed. The above was repeated ten times while the mixing conditions (matrix) were unchanged. Even though the recordings were unchanged, due to the iterative nature of ICA the outcome was different for each repetition. $|G|$ was normalized using Frobenius Norm to obtain $\|G\|$. Frobenius norm is widely used matrix norm and is based on the square root of the sum of squares. It can be computed by several equations such as

$$
\|A\|_{F}=\sqrt{\sum_{i=1}^{m} \sum_{j=1}^{n}\left|a_{i j}\right|^{2}}=\sqrt{\operatorname{trace}\left(A^{*} A\right)}=\sqrt{\sum_{i=1}^{\min (m, n)} \sigma_{i}^{2}},
$$

where $A^{*}$ denotes the conjugate transpose of $A, \sigma_{i}$ are the singular values of $A$, and the trace of a square matrix $A$ is the sum of the elements on the main diagonal. The Frobenius norm is submultiplicative and is very useful for numerical linear algebra. This norm is often easier to compute than induced norms and provides the most effective normalization values.

The values of the mixing equations parameters $\left(a_{i, j}\right)$ were in the range 0.4 to 0.9 and these values were changed for each repeat. These experiments were repeated for the three values of $M$ and for the conditions of independency $(N=$ $M)$, single dependency $(N=M-1)$, double dependency $(N=M-2)$, and total dependency cases $(N=1)$, with the simulation conditions shown in Table 1. Five sigma rule is indicative of high precision and was applied to identify the range $\|G\|$ corresponding to each condition. These values have been tabulated in Table 3 and were used to estimate $K$ for the second experiment.

Experiment 2. Experiments were conducted where $M$ number of recordings with $N$ number of independent sources were separated using FastICA. The experiments were conducted for $M=4,6$, and 8 and for $N=M$ (all independent) and $M-2$ (dependent). The global matrix was estimated using (1). The estimate of the numbers of ICs, $K$, was obtained based on $\|G\|$ (see Table 2 ), and $K-N=$ error in estimation was computed. The rank of the matrix, $R$, was also computed and compared with the value of $N$. 
TABLE 2: Average and standard deviation of $\|G\|$ for the four criterions.

\begin{tabular}{lcccc}
\hline Criterion & \multicolumn{3}{c}{ Average $\|G\|$} \\
& $M=4$ & $M=6$ & $M=8$ & Average \\
Independent & $0.558 \pm 0.024$ & $0.462 \pm 0.02$ & $0.38 \pm 0.016$ & 0.467 \\
Single dependency & $0.057 \pm 0.02$ & $0.048 \pm 0.002$ & $0.03 \pm 0.002$ & 0.045 \\
Double dependency & $0.0077 \pm 0.0007$ & $0.004 \pm 0.0001$ & $0.003 \pm 0.0002$ & 0.0049 \\
All dependent & $0.0007 \pm 7 \mathrm{E}-05$ & $0.0004 \pm 2 \mathrm{E}-05$ & $0.0003 \pm 1 \mathrm{E}-05$ & 0.000467 \\
\hline
\end{tabular}

Table 3: Normalized determinant conditions of global matrix for source dependency.

\begin{tabular}{lcc}
\hline Dependency levels & Source dependency & $\|G\|$ conditions \\
\hline 1 & $N=M$ (all independent sources) & $1>\|G\|>0.3$ \\
2 & $N=M-1$ (one dependent source) & $0.08>\|G\|>0.03$ \\
3 & $N=M-2$ (two dependent sources) & $0.008>\|G\|>0.003$ \\
4 & $N=1$ (all dependent sources) & $0.0008>\|G\|>0003$ \\
\hline
\end{tabular}

The next step was the random selection of $N$ out of the $M$ recordings. Experiments were conducted where the source separation using FastICA was done on the $M$ number of recordings and this was repeated with $N$ number of recordings. Signal to Interference Ratio (SIR) [6] was computed to determine the quality of separation. The average of the results of each repetition was computed and this has been reported.

\section{Results and Observations}

Experiment 1. The values of $\|G\|$ have been tabulated in Table 2 for all the four dependence categories and for $M=4$, 6 , and 8 . These values have been plotted on a log-scale plot in Figure 1. It is observed that as the dependency increases between sources, the value of $\|G\|$ decreases logarithmically for all $M(4,6$, and 8$)$.

Statistical analysis was conducted to determine the significance of the experimental outcomes. A result is statistically significant if it is unlikely to have occurred by chance. Statistical significance is the probability of incorrectly rejecting the null hypothesis (Type I error, or false positive determination). This decision can be made by testing the value of $P$ to be less than the significance level. $P$-values of .05 correspond to a $5 \%$ chance of incorrect rejection. While there is no definite rule for determining the threshold for the value of $P, .05$ is the most commonly used threshold for similar works and has been used in this research. The results of statistical analysis of the data indicate that the relationship between $M$ and $\|G\|$ is significant $(P<.05)$.

From Table 2 it is also observed that there is a small reduction in the value of $\|G\|$ with increase in $M$. Statistical analysis on each of the data sets demonstrates that this relationship is not significant $(P>.05)$. The range of $\|G\|$ values corresponding to the source dependency conditions based on the five-sigma rule has been tabulated in Table 3. These conditions were applied to the experiment 2 to estimate the $K$ values.
Results-Experiment 2. For the different values of $M$ and dependency conditions, error in the estimated value of ICs has been tabulated in Table 4. For comparison, the error based on rank of the matrix has also been tabulated. The error based on the proposed technique, $K-N$, error based on the rank of the matrix, $R-N$, average and standard deviation of the $\|G\|$ and SIR of the separated signals (in $\mathrm{dB}$ ) have been tabulated in Table 4(a). Table 4(a) has the results when ICA was performed on all $M$ recordings and $M>N$ and Table 4(b) has the results when ICA was performed on only $N$ randomly selected recordings. From Table $4(\mathrm{a})$ it is observed that estimate of $N$ based on $K$ resulted in an average error of 1, much smaller than error based on the rank of the matrix, $R$, which was 2.8. From Table 4(a), it is also observed that the average SIR value for $M$ recordings is $11.21 \mathrm{~dB}$ (range $9.1 \mathrm{~dB}$ to $13.8 \mathrm{~dB}$ ) and average $\|G\|$ is 0.02436 (SD $0.00195)$ when ICA was performed on all $M$ recordings and $M>N$.

From Table 4(b), it can be observed that when ICA is performed on $N$ recordings, there is a marked improvement in the quality of separation, and the SIR is $18.02 \mathrm{~dB}$ (range $17.32 \mathrm{~dB}$ to $19.84 \mathrm{~dB}$ ) and $\|G\|$ is 0.461 (SD 0.00048). The estimate of $N$ is not relevant in this table. From Tables 4(a) and $4(\mathrm{~b})$, it is also observed that the higher the quality of separation of the outcomes, the higher the value of $\|G\|$. While the value of $\|G\|$ was 0.004 when SIR was $9.1 \mathrm{~dB},\|G\|$ was 0.496 when SIR was $19.8 \mathrm{~dB}$.

\section{Conclusion}

Successful use of independent component analysis (ICA) to separate the independent components (ICs) requires the number of ICs to be prior determined. Without this information, the outcome of ICA can result in poor quality of separation. This paper has proposed and verified the use of $\|G\|$ ( $G$ is the global matrix generated by ICA) to identify the number of ICs in a given mixture. The results indicate that this method can also be used to determine the quality of separation. 
TABLE 4

(a) Error in estimated number of ICs using $\|G\|$, and using rank of the matrix, Mean and Standard deviation of $\|G\|$ and mean SIR when ICA is performed on all the $M$ recordings for different undercomplete situation.

\begin{tabular}{lccccc}
\hline$M$ & $N$ & $K-N$ & $R-N$ & $\|G\|$ & Mean SIR \\
\hline 4 & 2 & 0 & 2 & $0.0077 \pm 0.0007$ & $11 \mathrm{~dB}$ \\
6 & 4 & 0 & 2 & $0.0061 \pm 0.0001$ & $11.8 \mathrm{~dB}$ \\
6 & 2 & 2 & 2 & $0.004 \pm 0.00142$ & $9.1 \mathrm{~dB}$ \\
8 & 6 & 1 & $0.048 \pm 0.00043$ & $13.8 \mathrm{~dB}$ \\
8 & 4 & 2 & $0.056 \pm 0.0032$ & $10.34 \mathrm{~dB}$ \\
Average & 3.6 & 1 & 2.8 & $0.02436 \pm 0.00195$ & $11.21 \mathrm{~dB}$ \\
\hline
\end{tabular}

(b) Error in estimated number of ICs using $\|G\|$, and using rank of the matrix, Mean and Standard deviation of $\|G\|$ and mean SIR when ICA is performed on $N$ number of randomly selected recordings.

\begin{tabular}{lccccc}
\hline$M$ & $N$ & $K-N$ & $R-N$ & $\|G\|$ & Mean SIR \\
\hline 4 & 2 & 0 & 0 & $0.485 \pm 0.00012$ & $19 \mathrm{~dB}$ \\
6 & 4 & 0 & 0 & $0.452 \pm 0.00032$ & $18.51 \mathrm{~dB}$ \\
6 & 2 & 0 & 0 & $0.496 \pm 0.000273$ & $19.84 \mathrm{~dB}$ \\
8 & 6 & 0 & 0 & $0.384 \pm 0.00104$ & $17.32 \mathrm{~dB}$ \\
8 & 4 & 0 & 0 & $0.418 \pm 0.00016$ & $18.02 \mathrm{~dB}$ \\
Average & & 0 & & 0.461 & $18.54 \mathrm{~dB}$ \\
\hline
\end{tabular}

This paper has proposed a two-step approach of source separation when the number of sources is not known. The first step requires running ICA on the $M$ recordings and determining the number of ICs, $N$ in $M$ recordings based on determinant value of $G$. The second step is to run ICA again, this time on a random selection of $N$ out of the $M$ recordings. The results indicate a marked improvement of the quality of separation compared to using ICA on all $M$ recordings. This technique is not based on any assumptions regarding the number of ICs in the mixture. In the present form, this is based on the commonly held assumptions related to the applicability of ICA; that is, (i) the mixing matrix is linear and stationary and (ii) the noise level is low. This outcome of this research can be directly applied for blind source separation problems, where the number of sources is not known. The measure of quality of separation is very relevant in situations where it is difficult to estimate the quality of separation, such as in biosignal applications.

\section{References}

[1] P. Comon, "Independent component analysis, a new concept?" Signal Processing, vol. 36, no. 3, pp. 287-314, 1994.

[2] A. J. Bell and T. J. Sejnowski, "An information-maximization approach to blind separation and blind deconvolution," Neural Computation, vol. 7, no. 6, pp. 1129-1159, 1995.

[3] A. Hyvärinen, K. Karhunen, and E. Oja, Independent Component Analysis, John Wiley \& Sons, New York, NY, USA, 2001.

[4] J.-F. Cardoso, "Infomax and maximum likelihood for blind source separation," IEEE Signal Processing Letters, vol. 4, no. 4, pp. 112-114, 1997.

[5] C. J. James and C. W. Hesse, "Independent component analysis for biomedical signals," Physiological Measurement, vol. 26, no. 1, pp. R15-R39, 2005.
[6] A. Cichocki and S. Amari, Adaptive Blind Signal and Image Processing, John Wiley \& Sons, New York, NY, USA, 2002.

[7] S. Makeig, A. J. Bell, T. P. Jung, and T. J. Sejnowski, "Independent component analysis of electroencephalographic data," in Advances in Neural Informations Processing System, vol. 8, pp. 145-151, 2001.

[8] M. S. Lewicki and T. J. Sejnowski, "Learning overcomplete representations," Neural Computation, vol. 12, no. 2, pp. 337$365,2000$.

[9] T.-W. Lee, M. S. Lewicki, M. Girolami, and T. J. Sejnowski, "Blind source separation of more sources than mixtures using overcomplete representations," IEEE Signal Processing Letters, vol. 6, no. 4, pp. 87-90, 1999.

[10] P. Bofill and M. Zibulevsky, "Underdetermined blind source separation using sparse representations," Signal Processing, vol. 81, no. 11, pp. 2353-2362, 2001.

[11] S.-I. Amari, "Natural gradient learning for over- and undercomplete bases in ICA," Neural Computation, vol. 11, no. 8, pp. 1875-1883, 1999.

[12] M. Joho, H. Mathis, and R. H. Lambert, "Overdetermined blind source separation: using more sensors than source signals in a noisy mixture," in Proceedings of 2nd International Conference on Independent Component Analysis (ICA '02), pp. 81-86, Helsinki, Finland, June 2002.

[13] F. Vrins, J. A. Lee, M. Verleysen, V. Vigneron, and C. Jutten, "Improving independent component analysis performances by variable selection," in Proceedings of the 13th Workshop on Neural Networks for Signal Processing, pp. 359-368, 2003.

[14] J. V. Stone and J. Porrill, "Undercomplete independent component analysis for signal separation and dimension reduction," Tech. Rep., Department of Psychology, University of Shefield, 1998.

[15] C. D. Meyer, Matrix Analysis and Applied Linear Algebra, Cambridge University Press, Cambridge, UK, 2000.

[16] A. Hyvärinen, "Fast and robust fixed-point algorithms for independent component analysis," IEEE Transactions on Neural Networks, vol. 10, no. 3, pp. 626-634, 1999. 